\title{
Correlations between left atrial strain and left atrial pressures values in patients undergoing atrial fibrillation ablation
}

\author{
Beata Uziębło-Życzkowska, Paweł Krzesiński, Agnieszka Jurek, Krystian Krzyżanowski, Marek Kiliszek \\ Department of Cardiology and Internal Diseases, Military Institute of Medicine, Warszawa, Poland
}

\section{Editorial}

by Longobardo et al.

see p. 1195

\section{Correspondence to:}

Beata Uziębło-Życzkowska, MD, $\mathrm{PhD}$,

Department of Cardiology and Internal Diseases,

Military Institute of Medicine

Szaserów 128, 04-141 Warszawa, phone + 48226816389 ,

e-mail:

buzieblo-zyczkowska@wim.mil.pl Copyright by the Author(s), 2021

Kardiol Pol. 2021;

79 (11): 1223-1230

DOI: 10.33963/KP.a2021.0113

Received:

May 31, 2021

Revision accepted:

September 20, 2021

Published online:

September 20, 2021

\section{A B S T R A C T}

Background: We aimed to investigate the correlations between left atrial strain (LAS) assessed by speckle tracking echocardiography and directly measured left atrial pressures (LAP) values and to estimate the features of patients with lower LAS values.

Methods: A prospective, observational study was performed on patients with atrial fibrillation (AF) undergoing ablation. Detailed transthoracic echocardiography with the speckle tracking method was used to estimate the LAS reservoir (LASr) and direct measurements of LAP during ablation in all patients.

Results: A total of 172 patients were included (98 with sinus rhythm [SR] and 74 with AF). The patients with lower LASr (first tercile) compared to those with higher (third tercile), were older, more often female, presented with a larger left atrium (LA) (per the LA area and volume index), and had more impaired left ventricular (LV) diastolic function parameters (e', E/e'). The correlation analysis of the echocardiographic parameters with the LAP revealed the most significant correlations in the SR group, where the E/e' ratio, LASr, and LAS contraction (LASct) values were all associated with LAP. The cut-off value of LASr estimating high elevated mean LAP ( $\geq 15 \mathrm{~mm} \mathrm{Hg}$ ) was 21.88 (Area Under Curve [AUC], 0.81; 95\% confidence interval [CI], 0.72-0.90; $P<0.0001)$ for the SR group and 11.25 (AUC, $0.66 ; 95 \% \mathrm{Cl}, 0.53-0.80 ; P=0.016$ ) for the AF group.

Conclusions: AF patients with lower LASr are older, more often female, have a larger LA, and have more impaired LV diastolic function. Low LASr and LASct and high E/e' ratio values are associated with higher LAP in AF patients with SR.

Key words: atrial fibrillation, left atrial pressure, left atrial strain, speckle tracking echocardiography

Kardiol Pol 2021; 79, 11: 1223-1230

\section{INTRODUCTION}

The noninvasive assessment of left atrium (LA) function by echocardiography is important in patients with atrial fibrillation (AF) qualified for ablation [1-3]. Left atrial strain (LAS) analysis by speckle tracking echocardiography (STE) is a relatively new method for evaluating LA function [4-6]. Some reports have highlighted the role of LAS in predicting the recurrence of AF after ablation [7] and a relationship between LAS values and invasively estimated left atrial pressure (LAP) values [8, 9]. High LAP has proven to be an independent predictor of symptomatic AF recurrence after catheter ablation and has been associated with advanced LA remodeling [10].

This study aimed to investigate the relationship between LAS and LAP and estimate 
WHAT'S NEW?

The use of speckle tracking echocardiography to assess left atrial function is a particularly promising method in patients with atrial fibrillation. Our study aimed to present detailed characteristics of patients depending on the obtained values of left atrial longitudinal strain, as well as to investigate the relationship between left atrial strain values and left atrial pressure measured directly during ablation. We showed that patients with atrial fibrillation and lower left atrial reservoir strain values were older and women, more often, had a larger left atrium and more impaired left ventricular diastolic function. Low left atrial reservoir and contraction strain, and high E/e'ratio values are associated with higher left atrial pressures in patients with atrial fibrillation assessed during sinus rhythm.

the distinctive clinical and echocardiographic features of AF patients with lower LAS values.

\section{METHODS}

\section{Study design and patients}

This was a prospective, observational, single-center study based on data of consecutive AF patients who were admitted to a Polish cardiology center for the first-ever ablation of AF. Data about AF subtypes across the study cohort are included in Table 1. Patients were not receiving any antiarrhythmic therapy during the study. The data were collected from 2019 to 2020 . All patients had transthoracic and transesophageal echocardiography (TTE and TEE, respectively) in preparation for ablation. Patients who (1) had a thrombus in the left atrial appendage;

Table 1. Comparative analysis between lower (first tercile) and higher (third tercile) left atrial strain reservoir values within the atrial fibrillation group

\begin{tabular}{|c|c|c|c|c|c|}
\hline & \multicolumn{5}{|c|}{ AF group } \\
\hline & n & $1^{\text {st }}$ tercile, $\mathbf{n}=\mathbf{2 3}$ & $\mathbf{n}$ & $3^{\text {rd }}$ tercile, $n=23$ & P-value \\
\hline \multicolumn{6}{|l|}{ Demographic data } \\
\hline Age, years, median (IQR) & 23 & $69(67-73)$ & 23 & $64(60-69)$ & 0.046 \\
\hline Female gender, n (\%) & 23 & $14(60.9)$ & 23 & $4(17.4)$ & 0.006 \\
\hline Body mass index, $\mathrm{kg} / \mathrm{m}^{2}$, median (IQR) & 23 & $30.8(27-34)$ & 23 & $30.8(26-36)$ & 0.68 \\
\hline \multicolumn{6}{|l|}{ Echocardiographic data, median (IQR) } \\
\hline RVDd, mm & 23 & $28(27-33)$ & 23 & $30(28-32)$ & 0.75 \\
\hline IVSd, mm & 23 & $11(10-12)$ & 23 & $11(10-13)$ & 0.37 \\
\hline LVDd, mm & 23 & $51(44-54)$ & 23 & $47(45-50)$ & 0.10 \\
\hline LVMI, $\mathrm{g} / \mathrm{m}^{2}$ & 23 & $133.5(109-143)$ & 23 & $117.2(101-138)$ & 0.26 \\
\hline LVH, n (\%) & 23 & $10(43.5)$ & 23 & $13(56.5)$ & 0.56 \\
\hline $\mathrm{e}^{\prime}$ average, $\mathrm{cm} / \mathrm{s}$ & 23 & $7(6.5-9)$ & 23 & $9(9-10)$ & 0.004 \\
\hline E/e' average & 23 & $13.06(10-16)$ & 23 & $8.5(7.7-9.8)$ & 0.003 \\
\hline $\mathrm{LV} E F, \%$ & 23 & $56(46-63)$ & 23 & $59.5(52-63)$ & 0.37 \\
\hline $\mathrm{LAVI}, \mathrm{ml} / \mathrm{m}^{2}$ & 23 & $54.06(45-67)$ & 23 & $41.10(33.5-45)$ & $<0.001$ \\
\hline LAEF, \% & 22 & $28.02(20-33)$ & 23 & $31.25(28.5-40)$ & 0.10 \\
\hline LA enlargement, n (\%) & 23 & $23(100)$ & 23 & $17(73.9)$ & 0.021 \\
\hline \multicolumn{6}{|c|}{ Speckle tracking echocardiography data - left atrial and left ventricular function parameters } \\
\hline LASr, \%, mean (SD) & 23 & $7.443(1.817)$ & 23 & $15.164(1.783)$ & $<0.001$ \\
\hline LASct, \%, mean (SD) & & - & & - & \\
\hline LAScd, \%, mean (SD) & & - & & - & \\
\hline LV GLS, \%, median (IQR) & 21 & $12.4(10-16)$ & 22 & $15(13-17)$ & 0.09 \\
\hline \multicolumn{6}{|l|}{ Left atrial pressure, median (IQR) } \\
\hline LAP peak, $\mathrm{mm} \mathrm{Hg}$ & 23 & $31(19-42)$ & 23 & $19(17-25)$ & 0.007 \\
\hline LAP min, $\mathrm{mm} \mathrm{Hg}$ & 23 & $12(8-17)$ & 23 & $10.5(7-12)$ & 0.24 \\
\hline LAP mean, $\mathrm{mm} \mathrm{Hg}$ & 23 & $22(13-28)$ & 23 & $14(13-17)$ & 0.022 \\
\hline \multicolumn{6}{|l|}{ Clinical data, n (\%) } \\
\hline Heart failure & 23 & $10(43.5)$ & 23 & $4(17.4)$ & 0.11 \\
\hline Hypertension & 23 & $17(73.9)$ & 23 & $17(73.9)$ & 1 \\
\hline Transient ischemic attack/stroke & 23 & $2(8.7)$ & 23 & $3(13)$ & 1 \\
\hline Chronic kidney disease & 23 & $8(34.8)$ & 23 & $2(8.7)$ & 0.07 \\
\hline \multicolumn{6}{|l|}{ Laboratory data, median (IQR) } \\
\hline eGFR, $\mathrm{ml} / \mathrm{min} / 1.73 \mathrm{~m}^{2}$ & 23 & $90(54-90)$ & 23 & $90(90-90)$ & 0.11 \\
\hline
\end{tabular}

Abbreviations: AF, atrial fibrillation; CSI, contraction strain index; eGFR, estimated glomerular filtration rate; IQR, interquartile range; IVSd, interventricular septal dimension; $L A$, left atrial; LAEF, left atrial emptying fraction; LAP, left atrial pressure; LAScd, left atrial strain during conduit phase; LASct, left atrial strain during contraction phase; $L A S r$, left atrial strain during reservoir phase; LAV, left atrial volume; LAVI, left atrial volume index; LVDd, left ventricular end-diastolic dimension; LV EF, left ventricular ejection fraction; LV GLS, left ventricular global longitudinal strain; LVH, left ventricular hypertrophy; LVM, left ventricular mass; LVMI, left ventricular mass index; PA-TDI, total atrial conduction time; RVDd, right ventricular end-diastolic dimension; SD, standard deviation 
(2) had severe valvular heart disease; (3) were at the age of > 80 years; (4) had poor echocardiographic image quality were excluded from the study. No other exclusion criteria were used.

For all enrolled study subjects, we collected numerous data, including baseline demographic characteristics, type of AF, medical history and concomitant diseases, diagnostic test results, and information about oral anticoagulation therapy.

\section{Ethical approval}

The study was conducted according to good clinical practice guidelines and the Declaration of Helsinki. The study protocol was approved by the local ethics committee (50/WIM/2019), and each patient provided written consent.

\section{Standard transthoracic echocardiography}

The TTEs were performed directly before ablation using a high-quality echocardiograph (Vivid E95, General Electric, Boston, MA, USA). All examinations were analyzed offline by a single experienced echocardiographer (blinded to clinical status) accredited by the Section of Echocardiography of the Polish Cardiac Society. During the study, all the right ventricle and left ventricular (RV and LV) measurements, as well as LA measurements, were performed as recommended [11]. The LA emptying fraction (LAEF) was determined via the following formula: (LA maximum volume - LA minimum volume)/LA maximum volume $\times 100 \%$. The atrial conduction time (PA-TDI interval) was assessed as an interval between the initiation of the P-wave on the surface electrocardiogram and the peak of the A-wave on the tissue Doppler imaging of the side wall of the LA, just over the mitral annulus.

All echocardiographic measurements were conducted according to the current guidelines of the European and American Societies of Cardiology [11].

\section{Speckle tracking strain parameters}

Two-dimensional echocardiography was performed in a certified echocardiography laboratory using a General Electric Vivid E95 ultrasound system (1.5-4.5 MHz transducer). Measurements were made offline from recorded digital loops of three non-foreshortened apical chamber views (4AC, $2 A C$, and $3 A C$ ), ensuring a frame rate of 60-80 frames per second. Analyses of all strain parameters were performed offline in all studies with adequate quality via GE EchoPAC BT 12 software, as recommended in the Expert Consensus Statement [12]. The longitudinal strain was performed for the LV (LV global longitudinal strain, LVGLS) and LAS. All LAS parameters: LAS during the reservoir phase (LASr), LAS during the conduit phase (LAScd), and LAS during the contraction phase (LASct) were measured according to the current recommendations [13, 14]. LASct and LAScd were calculated only in the group of patients with sinus rhythm (SR) during echocardiogra- phy. For all strain measurements, while some segments were excluded due to the inability to achieve adequate tracking, global strain values were calculated by averaging the values measured in the remaining segments. The contraction strain index (CSI) was calculated by the formula CSI $=($ LASr $/$ LASct $) \times 100$.

Reproducibility of the strain measurements was assessed in 20 randomly selected patients, as described previously [15].

All measured LAS values turned out to be significantly lower in the group of patients with AF during echocardiography. For this reason, a comparative analysis was performed for two subgroups - the patients with SR and the patients with AF during TTE.

\section{Left atrial pressures measurements}

The LA was accessed through a double transseptal puncture using an 8.5 transseptal sheath, a Swartz ${ }^{\mathrm{TM}}$ SL0, and a BRK ${ }^{\mathrm{TM}}$ needle (Abbott Medica, Plymouth, MN, USA). The LAP was measured directly, soon after the transseptal puncture, via a transseptal sheath with a special converter for invasive blood pressure measurement (B. Braun Melsungen AG, Melsungen, Germany) and linked with the Philips Azurion image-guided therapy platform (Philips Medical Systems DMC GmbH, Hannover, Germany). Three values were measured automatically in the LA: highest (LAP max), lowest (LAP min), and mean (LAP mean).

\section{Statistical analysis}

Statistical analyses were conducted with Statistica 13.0 (StatSoft Inc., Tulsa, OK, USA). The distribution and normality of data were assessed with the Shapiro-Wilk test. Continuous variables were presented as means (standard deviation [SD]) or medians (interquartile range [IQR]: $1^{\text {st }}-3^{\text {rd }}$ quartile), whereas categorical variables were presented as absolute and relative values (percentages). In a comparative analysis, the student's t-test was used for normally distributed data, whereas the Mann-Whitney U-test was used for data with non-normal distribution. A chi-square test with the Yates correction was used for categorical variables. A two-tailed $p$-value of $<0.05$ was considered statistically significant. For comparison, the study population was divided into 3 terciles according to the distribution of LASr values and separately from the SR and AF groups, where the first tercile was for the subjects with the smallest values of LASr, and the third tercile was for the subjects with the highest values. The correlation of echocardiographic findings, including LAS parameters with LAP values, was analyzed separately for the SR and AF groups using Spearman's correlation analysis (with the $r_{s}$ correlation coefficient as the main analysis). A Receiver Operating Characteristic (ROC) curves with Area Under Curve (AUC) were calculated to estimate the cut-off value for LASr as a predictor of elevated mean LAP $(\geq 15 \mathrm{~mm} \mathrm{Hg})$ in the AF and SR group. 


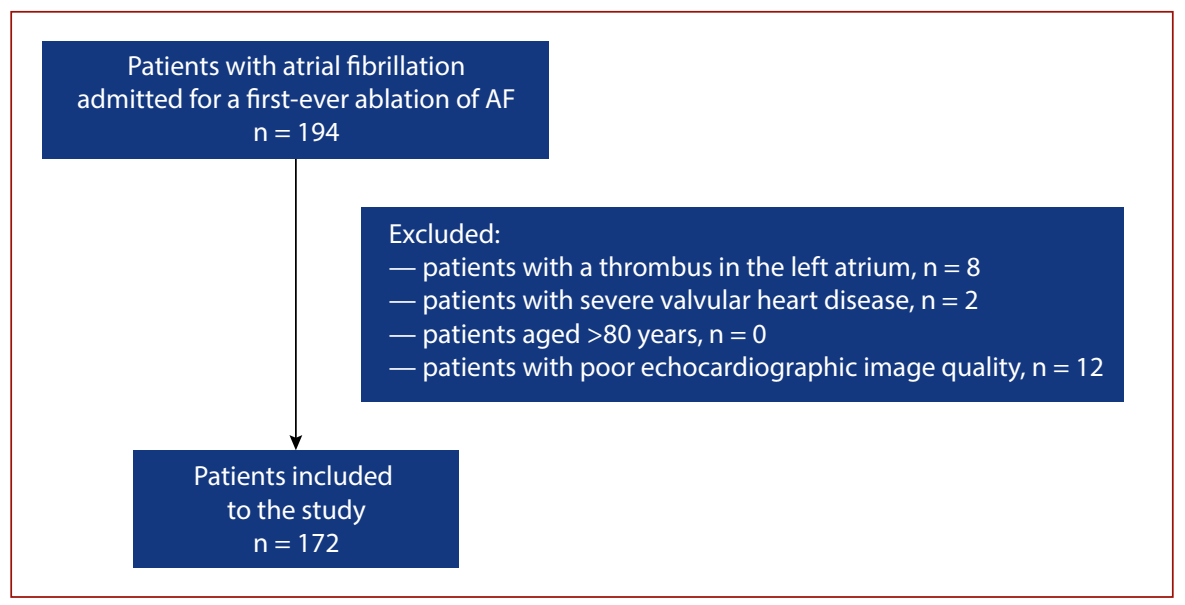

Figure 1. The flow chart of the study

Abbreviations: $\mathrm{AF}$, atrial fibrillation

\section{RESULTS}

\section{Baseline characteristics}

A total of 172 ablated AF patients were enrolled in the study (Figure 1). In total, 98 patients presented with SR during echocardiography, and 74 had AF. TTE and TEE were performed immediately prior to ablation, and the heart rhythm during both echocardiography examinations and ablation was the same in all enrolled patients. The baseline characteristics of the study population, including demographic, clinical, and echocardiographic data, are presented in Supplementary material, Table S1.

Patients in the AF group were compared to those in the SR group and did not differ in terms of clinical parameters. However, they were characterized by a significant enlargement of LA assessed by the LA volume index $(L A V I)$, reduced $L V$ systolic function assessed by $L V$ ejection fraction (LVEF) and LVGLS and had a more frequent occurrence of LV hypertrophy and lower LASr values. Moreover, analysis of LAS revealed lower values than those previously reported as normal (values reported as normal: $39 \%$ for LASr, $-17 \%$ for LASct, and $-23 \%$ for LAScd) [16] in both groups. Detailed LAS values are given in Supplementary material, Table S1. Mean and peak LAP, when measured directly during ablation, were significantly higher in the AF group.

\section{Characteristics of patients with lower LASr values}

In both assessed groups (AF and SR), the patients with lower LASr values (first tercile) were significantly older ( $P=0.046 ; P<0.0001$, respectively) and more often female $(P=0.006 ; P=0.01$, respectively). Moreover, patients in the first tercile in both groups, compared to those in the third tercile, had a significantly larger LA (assessed by LAVI) and more impaired diastolic LV function parameters by the $\mathrm{e}^{\prime}$ average value and the $\mathrm{E} / \mathrm{e}^{\prime}$ ratio.

The patients in the SR group also had more impaired systolic LV function (assessed by LVEF and LVGLS), more
LV muscle mass (assessed by LV mass [LVM] and the LVM index [LVMI]), lower LAEF, longer duration of PA-TDI, and significantly lower LASct and LAScd values. In addition, these patients had a significantly higher frequency of concomitant diseases, such as heart failure, hypertension, and vascular disease.

The remaining differences obtained in the comparison between both groups with lower LASr values are presented in Table 1 (AF) and Table 2 (SR).

No differences were found between the patients with lower and higher LASr values and clinical manifestation of AF (EHRA class).

The analysis of our population showed a negative correlation between age and all LAS values in the entire study group and the group with SR (Supplementary material, Table S2).

\section{Correlation of echocardiographic parameters with the LAP value}

The most prominent correlations were found in the SR group of patients between LASr and LASct values, as well as the E/e' ratio and LAP values. As for the results of the correlation analysis in the other groups (all groups and the AF group), the main differences were less noticeable, especially in the AF group. In the AF group, the most potent correlation was between the E/e' ratio and the LAPmax and LAPmean values. Detailed data are summarized in Tables 3 , 4 , and 5. In the SR group the cut-off value of LASr estimating elevated mean LAP ( $\geq 15 \mathrm{~mm} \mathrm{Hg}$ ) was 21.88 , with $71 \%$ sensitivity and $82 \%$ specificity (AUC, $0.81 ; 95 \% \mathrm{Cl}, 0.72-0.90$; $P<0.0001)$. In the AF group, the corresponding LASr cutoff value was 11.25 , with $72 \%$ sensitivity but $65.5 \%$ specificity (AUC, $0.66 ; 95 \% \mathrm{Cl}, 0.53-0.80 ; P=0.016$ ).

\section{DISCUSSION}

In this prospective, observational study, we investigated the clinical and echocardiographic characteristics of AF patients undergoing ablation. Long-term AF can lead to 
Table 2. Comparative analysis between lower (first tercile) and higher (third tercile) left atrial strain reservoir values within the sinus rhythm group

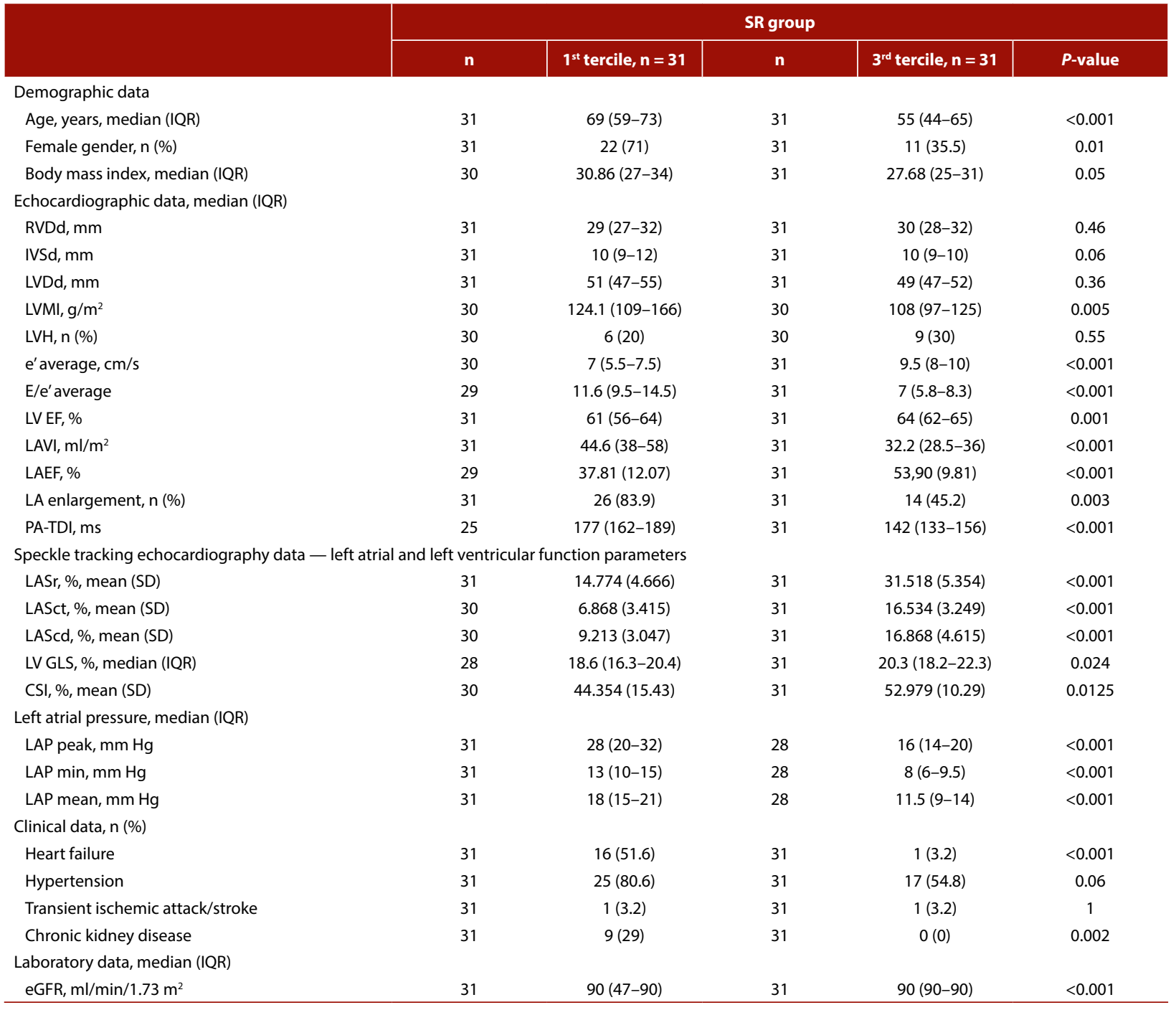

Abbreviations: SR, sinus rhythm; other — see Table 1

Table 3. Results of Spearman's correlation analysis of the entire study group

\begin{tabular}{l|c|c|c}
\multicolumn{1}{c|}{$\begin{array}{c}\text { Spearman's rank } \\
\text { correlation coefficient }\left(\mathbf{r}_{\mathbf{s}}\right)\end{array}$} & LAP max & LAP min & LAP mean \\
LASr & $-0.48^{\mathrm{b}}$ & $-0.36^{\mathrm{a}}$ & $-0.43^{\mathrm{a}}$ \\
LASct & $-0.55^{\mathrm{b}}$ & $-0.49^{\mathrm{b}}$ & $-0.52^{\mathrm{b}}$ \\
LAScd & $-0.25^{\mathrm{a}}$ & $-0.23^{\mathrm{a}}$ & $-0.23^{\mathrm{a}}$ \\
CSI & $-0.42^{\mathrm{a}}$ & $-0.31^{\mathrm{a}}$ & $-0.39^{\mathrm{a}}$ \\
LA area & $0.43^{\mathrm{a}}$ & $0.43^{\mathrm{a}}$ & $0.45^{\mathrm{a}}$ \\
LAVI & $0.42^{\mathrm{a}}$ & $0.40^{\mathrm{a}}$ & $0.41^{\mathrm{a}}$ \\
LAEF & $-0.46^{\mathrm{a}}$ & $-0.37^{\mathrm{a}}$ & $-0.44^{\mathrm{a}}$ \\
$\mathrm{e}^{\prime}$ average & $-0.30^{\mathrm{a}}$ & $-0.21^{\mathrm{a}}$ & $-0.26^{\mathrm{a}}$ \\
E/e' average & $0.59^{\mathrm{b}}$ & $0.33^{\mathrm{a}}$ & $0.48^{\mathrm{b}}$ \\
LV EF & $-0.21^{\mathrm{a}}$ & $-0.18^{\mathrm{a}}$ & $-0.19^{\mathrm{a}}$ \\
LV GLS & $-0.24^{\mathrm{a}}$ & $-0.26^{\mathrm{a}}$ & $-0.26^{\mathrm{a}}$ \\
\hline
\end{tabular}

${ }^{\mathrm{a}} P<0.05 ;{ }^{\mathrm{b}} P<0.001$

Abbreviations: see Table 1
Table 4. Results of Spearman's correlation analysis of the sinus rhythm group

\begin{tabular}{l|c|c|c}
\multicolumn{1}{c|}{$\begin{array}{c}\text { Spearman's rank } \\
\text { correlation coefficient }\left(\mathbf{r}_{\mathbf{s}}\right)\end{array}$} & LAP max & LAP min & LAP mean \\
LASr & $-0.49^{\mathrm{b}}$ & $-0.48^{\mathrm{b}}$ & $-0.47^{\mathrm{b}}$ \\
LASct & $-0.55^{\mathrm{b}}$ & $-0.49^{\mathrm{b}}$ & $-0.52^{\mathrm{b}}$ \\
LAScd & $-0.25^{\mathrm{a}}$ & $-0.23^{\mathrm{a}}$ & $-0.23^{\mathrm{a}}$ \\
CSI & $-0.42^{\mathrm{a}}$ & $-0.30^{\mathrm{a}}$ & $-0.39^{\mathrm{a}}$ \\
LA area & $0.43^{\mathrm{a}}$ & $0.43^{\mathrm{a}}$ & $0.43^{\mathrm{a}}$ \\
LAVI & $0.35^{\mathrm{a}}$ & $0.40^{\mathrm{a}}$ & $0.35^{\mathrm{a}}$ \\
LAEF & $-0.51^{\mathrm{b}}$ & $-0.38^{\mathrm{a}}$ & $-0.45^{\mathrm{a}}$ \\
GLS LV & -0.10 & -0.18 & -0.13 \\
$\mathrm{e}^{\prime}$ average & $-0.33^{\mathrm{a}}$ & $-0.24^{\mathrm{a}}$ & $-0.28^{\mathrm{a}}$ \\
E/e' average & $0.59^{\mathrm{b}}$ & $0.38^{\mathrm{b}}$ & $0.48^{\mathrm{b}}$ \\
LV EF & $-0.21^{\mathrm{a}}$ & -0.15 & -0.14 \\
\hline
\end{tabular}

${ }^{\mathrm{a}} P<0.05 ;{ }^{\text {b }} P<0.001$

Abbreviations: see Table 1 
Table 5. Results of Spearman's correlation analysis of the atrial fibrillation group

\begin{tabular}{l|c|c|c}
\multicolumn{1}{c|}{$\begin{array}{c}\text { Spearman's rank correlation } \\
\text { coefficient }\left(r_{s}\right)\end{array}$} & LAP max & LAP min & LAP mean \\
LASr & $-0.38^{\mathrm{a}}$ & -0.21 & $-0.35^{\mathrm{a}}$ \\
LA & $0.33^{\mathrm{a}}$ & $0.41^{\mathrm{a}}$ & $0.39^{\mathrm{a}}$ \\
LAVI & $0.36^{\mathrm{a}}$ & $0.34^{\mathrm{a}}$ & $0.37^{\mathrm{a}}$ \\
LAEF & $-0.32^{\mathrm{a}}$ & $-0.33^{\mathrm{a}}$ & $-0.32^{\mathrm{a}}$ \\
GLS LV & -0.04 & -0.23 & -0.12 \\
$\mathrm{e}^{\prime}$ average & $-0.45^{\mathrm{b}}$ & $-0.30^{\mathrm{a}}$ & $-0.39^{\mathrm{a}}$ \\
E/e' average & $0.56^{\mathrm{b}}$ & 0.23 & $0.45^{\mathrm{b}}$ \\
LV EF & -0.06 & -0.14 & -0.12 \\
\hline
\end{tabular}

${ }^{\mathrm{a}} P<0.05 ;{ }^{\mathrm{b}} P<0.001$

Abbreviations: see Table 1

structural, electrical, and functional LA remodeling. As a result, AF patients have an increased risk of mortality, thromboembolic events, stroke, and heart failure [16]. STE is well validated as a quantitative assessment tool for regional and global LA function $[17,18]$. This method makes it possible to detect early subclinical LA dysfunction in many cardiac diseases. The impairment of LASr can be detected in AF patients even before atrial dilatation has occurred. In the recent study, it was found, that LASr $>22 \%$ is a cut-off value for SR maintenance after catheter ablation [19]. In our study, all patients undergoing ablation had lower values of LAS than those previously reported as normal [16]. These results applied to patients with SR and those with AF during TTE. However, the LASr values were particularly lower in patients during AF. This occurrence prompted us to analyze both groups of patients separately. Kuppahally et al. [20] showed that LA wall fibrosis evaluated by delayed enhancement magnetic resonance imaging is inversely related to LAS values, and these are related to the AF burden. The reduced LAS value is considered an early non-invasive marker of the amount of LA wall fibrosis [21]. It is unclear, whether atrial fibrosis in $\mathrm{AF}$ patients is the result of $\mathrm{AF}$ itself or if it is related to other conditions, such as specific fibrotic atrial cardiomyopathy [22]. As experts point out, both mechanisms are possible and atrial arrhythmias can also induce atrial remodeling that contributes to the progression of AF [23]. Regardless of the mechanism, structural $A F$ remodeling is more frequent in patients with persistent AF. This can partly explain lower LAS values found in our patients with AF during TTE.

The comparative analysis of the 2 groups of AF patients (SR vs. AF groups) undergoing ablation also revealed that the patients from the AF group were characterized by not only significant enlargement of the LA but also by reduced LV systolic function and more frequent occurrence of LVH. In a group of $1483 \mathrm{AF}$ patients, Kuo et al. [24] demonstrated that impaired LV systolic function assessed by myocardial deformation (LVGLS, the strain rate) was common in patients with AF. Moreover, LV deformations measurements in AF patients provided an independent prognostic value over conventional echocardiographic measures with an improved risk prediction. In the mentioned study, $67.8 \%$ of the entire study cohort had abnormal LVGLS (defining the value of LVGLS $-14.7 \%$ as the abnormal GLS cut-off). Moreover, the patients with permanent AF had markedly lower GLS compared to those with paroxysmal AF $(-11.5 \%$ vs. $-14.4 \%)$. In our study, these values were better $(-14.1 \%$ vs. $19.7 \%$ ) for the AF and SR groups, respectively, but also more impaired in the AF group. Reduced LVGLS values in the group of patients with AF may worsen their prognosis. Dons et al. [25] reported that decreasing the GLS/ $\sqrt{ }(\mathrm{RR})$ interval ratio was associated with an increased risk of an adverse outcome in a group of 204 AF patients during 2.4 years of follow-up.

Per reports to date, reduced LAS values are strongly correlated with a worse prognosis for patients with AF. Therefore, an important result of our study is characterizing the group with the lowest LASr values as the group with the worst prognosis.

Characteristics of patients with the lowest LASr values, both assessed groups demonstrated that the patients with the lowest LASr values (first tercile) were significantly older and more often female ( $P=0.006 ; P=0.01$, respectively). Studies on the relationship of the LAS values with age did not provide unequivocal results. Previous studies have reported that the LASr value gradually decreases with age, regardless of the population studied, while the LASct values remain rather unchanged $[26,27]$. Most of the data show no significant difference between healthy men and women with respect to LA strain parameters [14]. However, studies conducted on patients with AF showed lower LASr values among women with AF [28].

Regarding the remaining parameters studied, the differences in the characteristics of the group with the lowest LAS values were mainly observed in the SR group. These patients were characterized by disturbances in both the structure (a larger LA, higher LVMI) and function (lower LVEF, LVGLS, LASct, LAScd, LAEF, e' average; higher E/e'; longer duration of PA-TDI) of the left heart chambers. In addition, these patients had a higher frequency of concomitant diseases, such as heart failure, hypertension, and vascular disease. Thus, this group is most likely to be at the highest risk of AF recurrence after ablation. However, confirmation requires follow-up studies.

Correlations between the LAS values and the LAP present another interesting issue. Individual LAS values reflect different phases of LA function and allow evaluation of phasic LA function throughout the cardiac cycle: LASr reflects LA compliance, LAcd as a conduit for LV filling, and LASct as a parameter of LA booster pump function. Likewise, LAP values are measured at different phases of LA function: LAPmax as the maximum height of the $v$ wave and LAPmin as the minimum of the $x$ trough during SR or the lowest value of the pressure curve during AF [29]. Although the greatest clinical utility concerns LASr in the case of echocardiography and LAPmean in the case of hemodynamic measurements, the importance of other LAS and LAP values is also increased. A study revealed that both LAPmax and 
LAPmean were significantly higher in patients with AF recurrence after catheter ablation [30]. In that study, in multivariate analysis, an LAPmax $>18 \mathrm{~mm} \mathrm{Hg}$ was significantly associated with an elevated risk for AF recurrence (hazard ratio $[H R], 3.8)$. Moreover, the LAPmax showed a better correlation with AF recurrence than the LAPmean (AUC LAPmax $=0.75$ vs. AUC LAPmean $=0.73$ ). Park et al. [10] also confirmed that LAPpeak during sinus rhythm was an independent predictor of clinical recurrence of AF (hazard ratio, $1.887 ; 95 \% \mathrm{Cl}, 1.063-3.350 ; P=0.028)$. Similar results were obtained in a study by Roh et al. [31], where LAPmax was compared with LA-late gadolinium enhancement extent on cardiac magnetic resonance imaging. Linhart et al. [32] revealed LAPmean was significantly higher in patients with recurrence of $A F(13.4 \pm 7.1$ vs. $11.0 \pm 5.2 \mathrm{~mm}$ $\mathrm{Hg} ; P=0.007)$. For all mentioned above reasons, we have decided to assess both echocardiographic parameters (LASvalues) and hemodynamic (LAPvalues) assessment of the LA function in its various phases.

In our study, the strongest correlations were found in the SR group, where LAP was correlated with LASr and LASct values as well as the E/e' ratio. These high LAP values measured during ablation proved to be independent predictors of clinical recurrence of $\mathrm{AF}$ and were associated with advanced LA remodeling [10]. It is difficult to explain weaker correlations between LAS and LAP values in the AF compared to the SR group. It is worth mentioning that Hewing et al. [33] obtained similar results. In patients with SR, LA reservoir, conduit, and contractile function are inversely correlated with hemodynamic parameters. However, in AF patients, there were no significant correlations between LA reservoir function and invasively obtained hemodynamic parameters.

In summary, the highlighted AF patients with the lowest LASr are older, more often female, have a larger $\mathrm{LA}$, and have more impaired LV diastolic parameters. The most prominent correlations were found in the SR group, where low LASr, low LASct, and high E/e' ratio values were associated with higher LAP.

\section{Clinical utility}

Direct measurement of LA pressures during ablation is an accurate but invasive method for assessing LA function. Non-invasive echocardiographic assessments of LA anatomy and function include more than volumetric and Doppler analyses. The use of the new STE method may be helpful, and it appears to be superior to conventional echocardiographic parameters of LA analysis [14, 15]. Assessment of LA function and structure has been used to predict both risks for thromboembolic events and the success of restoring SR after AF ablation [34, 35]. Characterizing the group of patients with the lowest LAS values may help predict the likelihood of SR maintenance after catheter ablation using LAS analysis. It would be quite valuable in selecting candidates for catheter ablation. High correlation of echocardiographic LA strain measurements with LA pressures could represent an attractive alternative approach for estimating hemodynamics in a group of AF people with SR.

\section{Limitations}

In the presented study, we assessed state-of-the-art strain parameters, and the measurements were performed by an experienced echocardiographer in accordance with the current recommendations. The main limitations were the small sample size and the lack of follow-up; thus, we can only assess the basic characteristics of the presented groups of patients and cannot assess whether the prognosis of the group with the lowest LAS values regarding the recurrence of arrhythmia is worse compared to other groups of patients.

\section{Supplementary material}

Supplementary material is available at https://journals. viamedica.pl/kardiologia_polska.

\section{Article information}

Conflict of interest: KK is a shareholder of Abbott. Other authors declare no conflict of interest.

Open access: This article is available in open access under Creative Common Attribution-Non-Commercial-No Derivatives 4.0 International (CC BY-NC-ND 4.0) license, allowing to download articles and share them with others as long as they credit the authors and the publisher, but without permission to change them in any way or use them commercially. For commercial use, please contact the journal office at kardiologiapolska@ptkardio.pl.

How to cite: Uziębło-Życzkowska B, Krzesiński P, Jurek A, et al. Correlations between left atrial strain and left atrial pressures values in patients undergoing atrial fibrillation ablation. Kardiol Pol. 2021; 79(11): 1223-1230, doi: 10.33963/KP.a2021.0113.

\section{REFERENCES}

1. Vieira MJ, Teixeira R, Gonçalves L, et al. Left atrial mechanics: echocardiographic assessment and clinical implications. J Am Soc Echocardiogr. 2014; 27(5): 463-478, doi: 10.1016/j.echo.2014.01.021, indexed in Pubmed: 24656882.

2. Inciardi RM, Giugliano RP, Claggett B, et al. ENGAGE AF-TIMI 48 Investigators. Left atrial structure and function and the risk of death or heart failure in atrial fibrillation. Eur J Heart Fail. 2019; 21(12): 1571-1579, doi: 10.1002/ejhf.1606, indexed in Pubmed: 31777160.

3. Gupta S, Matulevicius SA, Ayers CR, et al. Left atrial structure and function and clinical outcomes in the general population. Eur Heart J. 2013; 34(4): 278-285, doi: 10.1093/eurheartj/ehs188, indexed in Pubmed: 22782941.

4. Frydas A, Morris DA, Belyavskiy E, et al. Left atrial strain as sensitive marker of left ventricular diastolic dysfunction in heart failure. ESC Heart Fail. 2020; 7(4): 1956-1965, doi: 10.1002/ehf2.12820, indexed in Pubmed: 32613770.

5. Jasic-Szpak E, Marwick TH, Donal E, et al. Prediction of AF in heart failure with preserved ejection fraction: incremental value of left atrial strain. JACC Cardiovasc Imaging. 2021; 14(1): 131-144, doi: 10.1016/j. jcmg.2020.07.040, indexed in Pubmed: 33413883.

6. Sahebjam M, Mazareei A, Lotfi-Tokaldany $M$, et al. Comparison of left atrial function between hypertensive patients with normal atrial size and normotensive subjects using strain rate imaging technique. Arch Cardiovasc Imag. 2014; 2(1), doi: 10.5812/acvi.16081.

7. Yasuda R, Murata $M$, Roberts $R$, et al. Left atrial strain is a powerful predictor of atrial fibrillation recurrence after catheter ablation: study of a heterogeneous population with sinus rhythm or atrial fibrillation. 
Eur Heart J Cardiovasc Imaging. 2015; 16(9): 1008-1014, doi: 10.1093/ehjci/jev028, indexed in Pubmed: 25750193.

8. Cameli M, Sparla S, Losito M, et al. Correlation of left atrial strain and doppler measurements with invasive measurement of left ventricular end-diastolic pressure in patients stratified for different values of ejection fraction. Echocardiography. 2016; 33(3): 398-405, doi: 10.1111/echo.13094, indexed in Pubmed: 26493278.

9. Lindqvist $P$, Henein $M$. Left atrial strain rate during atrial contraction predicts raised pulmonary capillary wedge pressure: evidence for left atrio-ventricular interaction. Int J Cardiovasc Imaging. 2021; 37(5): 15291538, doi: 10.1007/s10554-020-02126-7, indexed in Pubmed: 33392878.

10. Park J, Joung B, Uhm JS, et al. High left atrial pressures are associated with advanced electroanatomical remodeling of left atrium and independent predictors for clinical recurrence of atrial fibrillation after catheter ablation. Heart Rhythm. 2014; 11(6): 953-960, doi: 10.1016/j. hrthm.2014.03.009, indexed in Pubmed: 24607916.

11. Lang RM, Badano LP, Mor-Avi V, et al. Recommendations for cardiac chamber quantification by echocardiography in adults: an update from the American Society of Echocardiography and the European Association of Cardiovascular Imaging. J Am Soc Echocardiogr. 2015; 28(1): 1-39. e14, doi: 10.1016/j.echo.2014.10.003, indexed in Pubmed: 25559473.

12. Mor-Avi V, Lang RM, Badano LP, et al. Current and evolving echocardiographic techniques for the quantitative evaluation of cardiac mechanics: ASE/EAE consensus statement on methodology and indications endorsed by the Japanese Society of Echocardiography. J Am Soc Echocardiogr. 2011; 24(3): 277-313, doi: 10.1016/j.echo.2011.01.015, indexed in Pubmed: 21338865.

13. Badano LP, Kolias TJ, Muraru D, et al. Industry representatives, Reviewers: This document was reviewed by members of the 2016-2018 EACVI Scientific Documents Committee. Standardization of left atrial, right ventricular, and right atrial deformation imaging using two-dimensional speckle tracking echocardiography: a consensus document of the EACVI/ASE/Industry Task Force to standardize deformation imaging. Eur Heart J Cardiovasc Imaging. 2018; 19(6): 591-600, doi: 10.1093/ehjci/jey042, indexed in Pubmed: 29596561.

14. Pathan $F, D^{\prime}$ Elia N, Nolan MT, et al. Normal ranges of left atrial strain by speckle-tracking echocardiography: a systematic review and meta-analysis. J Am Soc Echocardiogr. 2017; 30(1):59-70.e8, doi: 10.1016/j. echo.2016.09.007, indexed in Pubmed: 28341032.

15. Uziebło-Życzkowska B, Krzesiński P. Correlations between left ventricular and left atrial function assessed by speckle tracking echocardiography in patients with treated well-controlled arterial hypertension. Cardiol Res Practice. 2021, doi: https://doi.org/10.1155/2021/6674081.

16. Hindricks G, Potpara T, Dagres N, et al. ESC Scientific Document Group. 2020 ESC Guidelines for the diagnosis and management of atrial fibrillation developed in collaboration with the European Association for Cardio-Thoracic Surgery (EACTS): The Task Force for the diagnosis and management of atrial fibrillation of the European Society of Cardiology (ESC) Developed with the special contribution of the European Heart Rhythm Association (EHRA) of the ESC. Eur Heart J. 2021;42(5): 373-498, doi: 10.1093/eurheartj/ehaa612, indexed in Pubmed: 32860505.

17. Vianna-Pinton R, Moreno CA, Baxter CM, et al. Two-dimensional speckle-tracking echocardiography of the left atrium: feasibility and regional contraction and relaxation differences in normal subjects. J Am Soc Echocardiogr. 2009; 22(3): 299-305, doi: 10.1016/j.echo.2008.12.017, indexed in Pubmed: 19258177.

18. Cameli M, Caputo M, Mondillo S, et al. Feasibility and reference values of left atrial longitudinal strain imaging by two-dimensional speckle tracking. Cardiovasc Ultrasound. 2009; 7:6, doi: 10.1186/1476-7120-7-6, indexed in Pubmed: 19200402.

19. Liżewska-Springer A, Dąbrowska-Kugacka A, Lewicka E, et al. Biatrial strain as a new predictive marker of successful pulmonary vein ablation in patients with atrial fibrillation and preserved left ventricular function. Kardiol Pol. 2019; 77(4): 471-474, doi: 10.5603/KP.a2019.0016, indexed in Pubmed: 31025652.
20. Kuppahally SS, Akoum N, Burgon NS, et al. Left atrial strain and strain rate in patients with paroxysmal and persistent atrial fibrillation: relationship to left atrial structural remodeling detected by delayed-enhancement MRI. Circ Cardiovasc Imaging. 2010; 3(3): 231-239, doi: 10.1161/CIRCIMAGING.109.865683, indexed in Pubmed: 20133512.

21. Longobardo L, Todaro MC, Zito C, et al. Role of imaging in assessment of atrial fibrosis in patients with atrial fibrillation: state-of-the-art review. Eur Heart J Cardiovasc Imaging. 2014; 15(1): 1-5, doi: 10.1093/ehjci/jet116, indexed in Pubmed: 23798579.

22. Calkins H, Hindricks G, Cappato R, et al. 2017 HRS/EHRA/ECAS/APHRS/ /SOLAECE expert consensus statement on catheter and surgical ablation of atrial fibrillation. EP Europace. 2017; 20(1): e1-e160, doi: 10.1093/europace/eux274.

23. Goette A, Kalman J, Aguinaga L, et al. EHRA/HRS/APHRS/SOLAECE expert consensus on atrial cardiomyopathies: definition, characterization, and clinical implication. Europace. 2016; 18(10): 1455-1490, doi: 10.1093/europace/euw161.

24. Kuo JY, Chang SH, Sung KT, et al. Left ventricular dysfunction in atrial fibrillation and heart failure risk. ESC Heart Fail. 2020 [Epub ahead of print]; 7(6):3694-3706, doi: 10.1002/ehf2.12920, indexed in Pubmed: 32929859.

25. Dons M, Jensen JS, Olsen FJ, et al. Global longitudinal strain corrected by RR-interval is a superior echocardiographic predictor of outcome in patients with atrial fibrillation. Int J Cardiol. 2018; 263: 42-47, doi: 10.1016/j.ijcard.2018.02.038, indexed in Pubmed: 29754921.

26. Sun JP, Yang Y, Guo R, et al. Left atrial regional phasic strain, strain rate and velocity by speckle-tracking echocardiography: normal values and effects of aging in a large group of normal subjects. Int J Cardiol. 2013; 168(4): 3473-3479, doi: 10.1016/j.ijcard.2013.04.167, indexed in Pubmed: 23706316.

27. Meel $R$, Khandheria BK, Peters $F$, et al. Effects of age on left atrial volume and strain parameters using echocardiography in a normal black population. Echo Res Pract. 2016; 3(4): 115-123, doi: 10.1530/ERP-16-0038, indexed in Pubmed: 27884828.

28. Yoshida K, Obokata M, Kurosawa K, et al. Effect of sex differences on the association between stroke risk and left atrial anatomy or mechanics in patients with atrial fibrillation. Circ Cardiovasc Imaging. 2016; 9(10): e004999, doi: 10.1161/CIRCIMAGING.116.004999, indexed in Pubmed: 27729360.

29. Mark JB. Atlas of cardiovascular monitoring. Churchill Livingstone, New York 1998: Chapter 4.

30. Bergau L, Vollmann D, Luthje L, et al. Measurement of left atrial pressure is a good predictor of freedom from atrial fibrillation. Indian Pacing Electrophysiol J. 2014; 14(4): 181-193, doi: 10.1016/50972-6292(16)307744, indexed in Pubmed: 25057219.

31. Roh SY, Lee DI, Hwang SH, et al. Association of left atrial pressure with late gadolinium enhancement extent in patient who underwent catheter ablation for atrial fibrillation. Sci Rep. 2020; 10(1): 16486, doi: 10.1038/s41598-020-72929-0, indexed in Pubmed: 33020516.

32. Linhart $M$, Lewalter $T$, Mittmann-Braun EL, et al. Left atrial pressure as predictor for recurrence of atrial fibrillation after pulmonary vein isolation. J Interv Card Electrophysiol. 2013; 38(2): 107-114, doi: 10.1007/s10840-013-9803-9, indexed in Pubmed: 23793444.

33. Hewing B, Theres L, Spethmann S, et al. Left atrial strain predicts hemodynamic parameters in cardiovascular patients. Echocardiography. 2017; 34(8): 1170-1178, doi: 10.1111/echo.13595, indexed in Pubmed: 28664601.

34. Gupta DK, Giugliano RP, Ruff CT, et al. Effective Anticoagulation with Factor Xa Next Generation in AF-Thrombolysis in Myocardial Infarction 48 (ENGAGE AF-IMI 48) Echocardiographic Study Investigators. The prognostic significance of cardiac structure and function in atrial fibrillation: the ENGAGE AF-TIMI 48 echocardiographic substudy. J Am Soc Echocardiogr. 2016; 29(6):537-544, doi: 10.1016/j.echo.2016.03.004, indexed in Pubmed: 27106009.

35. Motoki H, Negishi K, Kusunose K, et al. Global left atrial strain in the prediction of sinus rhythm maintenance after catheter ablation for atrial fibrillation. J Am Soc Echocardiogr. 2014; 27(11): 1184-1192, doi: 10.1016/j.echo.2014.08.017, indexed in Pubmed: 25260436. 